Note

\title{
A new proposal regarding the transport mechanism of mercury in biological membranes
}

\author{
Marcantonio Bragadin ${ }^{\mathrm{a}, *}$, Sabrina Manente ${ }^{\mathrm{a}}$, Francesca Cima ${ }^{\mathrm{b}}$ \\ a Dipartimento di Scienze Ambientali, Università di Venezia, DD 213730123 Venice, Italy \\ ${ }^{\mathrm{b}}$ Dipartimento do Biologia, Via Ugo Bassi 58/b, Università di Padova, 35121 Padova, Italy \\ Received 28 January 2004; accepted 15 September 2004 \\ Available online 7 February 2005
}

\begin{abstract}
The interactions of mercury $\left(\mathrm{Hg}^{2+}\right)$ with biological membranes have been investigated. The experimental results indicate that $\mathrm{Hg}^{2+}$ induces a rapid alkalinization in energized Lysosomes from rat liver. The interpretation of the process is that the mercury enters the Lysosomes as a $\mathrm{Hg}(\mathrm{OH})_{2}$ electroneutral compound, thus inducing alkalinization in the matrix.
\end{abstract}

(C) 2004 Elsevier B.V. All rights reserved.

Keywords: Lysosomes; Mercury; Transport

\section{Introduction}

The interactions of $\mathrm{Hg}^{2+}$ with biological systems have been widely studied, as the compound is very toxic [1-3]. The results indicate that $\mathrm{Hg}^{2+}$ interacts with many proteins, in particular with the sulfur groups $[4,5]$ and uncouples the oxidative phosphorylation in mitochondria [3]. However, the molecular causes responsible for the toxic effects in whole organisms have never been explained. The aim of this study is not to give new elements as an explanation for the toxicity of mercury, but to clarify the transport mechanism of $\mathrm{Hg}^{2+}$ within the biological membranes since the transport mechanism would play an essential and propaedeutic part in any subsequent toxicological studies. The above problem was examined regarding liposomes in a previous article [6]. The experimental results indicate that $\mathrm{Hg}^{2+}$ behaves as a $\mathrm{Cl}^{-} / \mathrm{OH}^{-}$exchanger by means of an eletroneutral process. These results indicate that the $\mathrm{Hg}(\mathrm{OH})_{2}$

\footnotetext{
${ }^{*}$ Corresponding author. Tel.: +39 41 2348507/257 8511; fax: +39 41 $2348584 / 2578584$

E-mail address: bragadin@unive.it (M. Bragadin).
}

and $\mathrm{HgCl}_{2}$ compounds are transported through the membrane. In this paper, we have examined the $\mathrm{Hg}^{2+}$ transport system in Lysosomes extracted from rat liver. The results are in agreement with those found in artificial membranes [6], as the experimental evidence suggests the transport of $\mathrm{Hg}(\mathrm{OH})_{2}$ through the membrane.

\section{Materials and methods}

Lysosomes from rat liver have been prepared following the procedure described by Savant et al. [7]. The protein concentration has been determined by the Lowry method [8]. The lysosomes, after the last centrifugation of the preparation, have been resuspended in a medium containing: $0.1 \mathrm{M}$ sucrose, $50 \mathrm{mM} \mathrm{K} \mathrm{SO}_{4}, 20 \mathrm{mM}$ Hepes, pH 7.4, $2.5 \mathrm{mM} \mathrm{MgSO}_{4}$ and $500 \mu \mathrm{M}$ EGTA and stored at $0{ }^{\circ} \mathrm{C}$. The protein concentration of the resuspended lysosomes was $20 \mathrm{mg} / \mathrm{ml}$. The dye acridine orange (AO), $1 \mathrm{mM}$, was used in ethanol. AO uptake by lysosomes was followed spectrophotometrically at $20^{\circ} \mathrm{C}$ by a Jenway 6400 spectrophotometer at $492 \mathrm{~nm}$. After addition of $20 \mu \mathrm{l}$ lysosomes and $20 \mu \mathrm{l}$ of the dye AO 
before the addition of $2 \mathrm{mM}$ ATP, to the medium ( 2.5 $\mathrm{ml})$ the instrument was calibrated at zero absorbance.

All reagents were of analytical grade. 2,4-dinitrophenol (DNP), AO Mercury acetate, and bafilomycin were supplied by Fluka.

\section{Results and discussion}

The $\mathrm{pH}$ in the matrix is about 4 , in energized lysosomes, as a consequence of three factors:

- The preexistence of a $\mathrm{pH}$ of 5.2 (before the addition of ATP) due to a Donnam effect [9].

- The presence of an ATP-driven proton pump (Vacuolar ATPase) [10-13].

- The presence of a selective $\mathrm{Cl}^{-}$channel which, in a chloride medium, allows for the uptake of chloride ions in the matrix [10-12].

The driving force for the acidification of energized lysosomes is the vacuolar proton pump, which by utilizing the ATP energy, pumps the protons into the matrix. As the protons are positively charged, the formation of a positive-inside potential in the membrane would seem to be indicated here. The presence of this potential does not allow for an acidification of below $\mathrm{pH} 5$, as that potential is a force which opposes the influx of the positively charged protons. However, as a consequence of the presence of the selective chloride channel and $\mathrm{Cl}^{-}$influx from the medium, the potential inside the lysosomes is low (about $+40 \mathrm{mV}$ [10]), and under those conditions, the $\mathrm{pH}$ in the matrix is acidic (about 4).

The $\mathrm{pH}$ changes in lysosomes are generally followed by means of the spectral changes of weak permeant bases, such as acridine orange (AO) $[11,14]$. AO, following the mechanism of weak permeant bases [14], crosses the lysosomal membrane as an electroneutral compound and accumulates inside as the $\mathrm{pH}$ is acidic. The accumulation gives rise to a metachromatic effect, with a consequent chemical shift [15]. As a consequence, by operating at $492 \mathrm{~nm}$, the maximum of absorbance of the $\mathrm{AO}$ in water, the uptake of the $\mathrm{AO}$ is accompanied by an absorbance decrease. Fig. 1 shows an example of this experimental situation. Before the ATP addition, the AO absorbance decrease results from its accumulation in the matrix, where the $\mathrm{pH}$ is about 5.2. The addition of the ATP causes a further absorbance quenching up to a steady state in which the $\mathrm{pH}$ is about 4 .

In steady state, the addition of Bafilomycin, the inhibitor of the vacuolar ATPase [16], causes a slow alkalinization of the matrix (Fig. 1(a)), while the addition of an uncoupler (or protonophore) such as 2,4-dinitrophenol (DNP), causes a fast alkalinization of the matrix (Fig. 1(b)) at low doses. This effect is brought about by the classical uncoupling mechanism of DNP

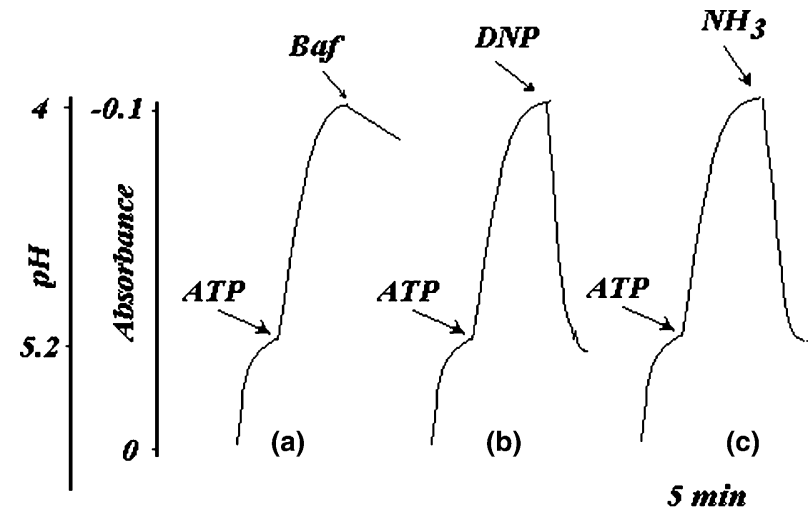

Fig. 1. The acidification of lysosomes followed by means of the AO dye and the effects of bafilomycin, DNP and Ammonia on the internal pH. Medium composition: $125 \mathrm{mM} \mathrm{KCl}, 10 \mathrm{mM}$ Hepes-Mops, $\mathrm{pH} 7.4$, $10 \mathrm{mM} \mathrm{MgCl}_{2}$, and $8 \mu \mathrm{M}, \mathrm{AO}$. The lysosomes were added to the medium and the final concentration was $0.2 \mathrm{mg} / \mathrm{ml}$. Under these conditions, before the addition of the ATP, the steady state corresponded to a $\mathrm{pH}$ of 5.2. After the ATP addition $(2 \mathrm{mM})$, the new steady state corresponded to a $\mathrm{pH}$ of about 4 . Under these conditions, the addition of $50 \mathrm{nM}$ bafilomycin (Baf) (a) gave rise to a slow alkalinization, whereas the addition of $100 \mu \mathrm{M}$ DNP (b) or 10 $\mathrm{mM}$ ammonia (in the form of ammonium chloride) (c) gave rise to a rapid alkalinization.

[17]: the compound enters as an anion (phenate), as the driving force is the positive-inside potential, and is extruded as an electroneutral undissociated compound, as the $\mathrm{pH}$ is acidic inside. Therefore, the mechanism is cyclic and during any cycle, a proton is extruded from the matrix (in the absence of an uncoupler or protonophore, the membrane is not permeant to the protons). This cyclic mechanism clearly indicates that low doses are necessary to induce alkalinization in the matrix. It should be noted that all uncouplers must be weak acids (or weak bases), because the undissociated form is present only in the weak acids (or weak bases). In the case where the uncoupler is a weak base, the cyclic mechanism is due to the uptake of the neutral form and to extrusion of the cation.

Analogously, a weak permeant base, such as ammonia, if added in steady state in a concentration above millimolar, causes a rapid alkalinization (Fig. 1(c)). In this case, the mechanism of the permeant weak base involves the accumulation of the undissociated compound in the matrix (as the acidic $\mathrm{pH}$ is the driving force), but, unlike the uncoupler, the cation (ammonium ion) is not extruded from the matrix [14]. This mechanism is not cyclic, and this explains why, in this case, the doses of weak base necessary to obtain the alkalinization are in the range of millimolar, whereas as regards the uncouplers they are in the order of micromolar.

Fig. 2(a) illustrates the rapid alkalinization of lysosomes when mercury $\left(\mathrm{Hg}(\text { acetate })_{2}\right)$ is added in steady state. The alkalinization rate is very rapid when compared with that induced by bafilomycin. This behaviour 


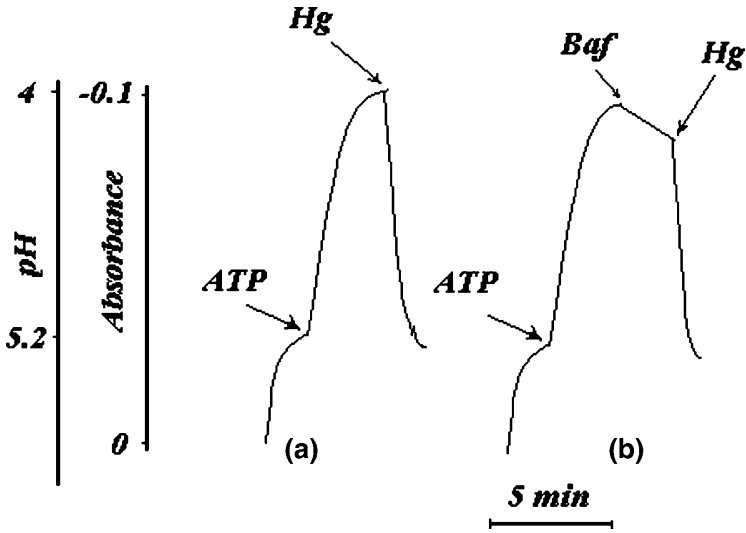

Fig. 2. The rapid alkalinization induced by mercury in energized lysosomes, both in the absence, and in the presence of bafilomycin. 10 $\mu \mathrm{M}$ mercury (in the form of acetate) was added to the energized lysosomes, first in the absence (a) and then in the presence (b) of bafilomycin. The medium and the conditions were the same as those in Fig. 1.

suggests that the effect of mercury is different from that brought about by the ATPase inhibitors. The above is confirmed by the experiments in Fig. 2(b), where the compound was added after the bafilomycin, i.e., when the ATPase was completely inhibited.

We conclude that the rapid alkalinization induced by the mercury, in a steady state, after energization, could be due to one of the following: an uncoupling mechanism, a weak base effect, or an inhibition of the chloride channel (but not due to an inhibition of the ATPase).

With regard to this last point, Fig. 3 shows an experiment in which the lysosomes are resuspended in a chloride-free medium. Under these conditions, the internal acidification is not brought about by the influx of a negative ion $\left(\mathrm{Cl}^{-}\right)$, but by the efflux of a $\left(K^{+}\right)$positive ion from the matrix, in the presence of the potassium carrier
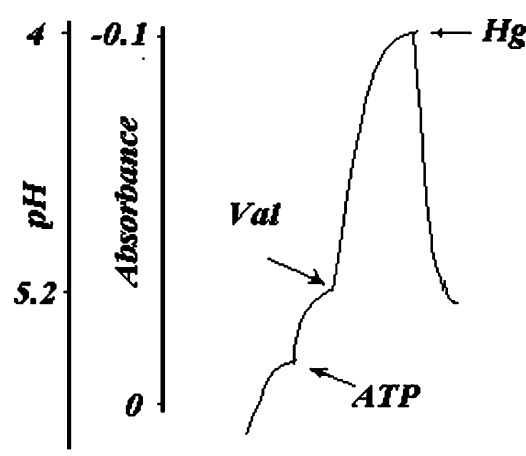

\section{$5 \mathrm{~min}$}

Fig. 3. The rapid alkalinization induced by mercury in energized lysosomes, in a chloride-free medium. Medium composition: $80 \mathrm{mM}$ $\mathrm{K}_{2} \mathrm{SO}_{4}, 10 \mathrm{mM}$ Hepes-Mops, $\mathrm{pH} 7.4,10 \mathrm{mM} \mathrm{MgSO}_{4}$, and $8 \mu \mathrm{M} \mathrm{AO}$. The lysosomes were added to the resuspending medium and the final concentration was $0.2 \mathrm{mg} / \mathrm{ml}$. After the addition of $2 \mathrm{mM} \mathrm{ATP}$, and Valinomycin (Val) $(10 \mu \mathrm{M}), 10 \mu \mathrm{M} \mathrm{Hg}$ (in the form of $\mathrm{Hg}(\text { acetate })_{2}$ ) was added in a steady state. valinomycin (the lysosomal matrix contains about 0.1 $\left.\mathrm{mM} K^{+}[10]\right)$. The addition of Mercury in a steady state induces a rapid alkalinization similar to that where $\mathrm{Cl}^{-}$ is present in the medium. This behavior supports the hypothesis that the $\mathrm{Cl}^{-}$channel is not influent in the transport mechanism.

The following two possibilities remain: i.e., the alkalinization induced by the mercury is due to either weak or an uncoupling effect.

The doses of $\mathrm{Hg}$ (acetate) $)_{2}$ were very low (around micromolar) and this can be interpreted to be the result of a cyclic uncoupling effect and not of a weak base effect.

However, the uncoupling effect would indicate the uptake of the electroneutral $\mathrm{Hg}(\mathrm{OH})_{2}$ compound and the subsequent extrusion of $\mathrm{Hg}^{2+}$ or $\mathrm{HgOH}^{+}$, but this mechanism is not probable since the $\mathrm{Hg}^{2+}$, once inside, strongly binds to the sulfur in the proteins.

If the alkalinization is due to a weak base effect, the uptake of $\mathrm{Hg}(\mathrm{OH})_{2}$ (as in the case of the uncoupling effect) occurs, but not the extrusion of the charged ion $\left(\mathrm{Hg}^{2+}\right.$ or $\left.\mathrm{HgOH}^{+}\right)$. In this case, the accumulation of $\mathrm{Hg}^{2+}$ inside the lysosomes is probably accompanied by its binding to the sulfur of the proteins with new uptake of $\mathrm{Hg}(\mathrm{OH})_{2}$ from the external medium. Therefore, as a consequence of this uptake of $\mathrm{Hg}(\mathrm{OH})_{2}$ from the medium, a strong alkalinization occurs, even if the doses of mercury are low if compared to those employed with weak permeant bases such as ammonia.

A similar behavior was seen to occur regarding artificial vesicles, where the addition of nanomolar quantities of $\mathrm{Hg}(\mathrm{OH})_{2}$ induced $\mathrm{pH}$ changes inside the liposomes [6].

To sum up, even though it is not possible, at the moment, to distinguish between the two mechanisms, we believe that the most probable behavior is one which is similar to that of weak bases but, whether this process depends on a weak base or on an uncoupling mechanism, the experiments in this paper should demonstrate that mercury crosses the biological membranes and, in particular, that the transport through the membrane occurs as (electroneutral) $\mathrm{Hg}(\mathrm{OH})_{2}$ compound. If this mechanism is found to be the correct one, the involvement of a physiological carrier, which has been postulated, but never identified, is not necessary to explain the presence of mercury inside the cells, since the mercury enters the phospholipidic bilayer in a similar manner to that concerning artificial vesicles. Obviously, this mechanism does not exclude the possibility that other species of mercury can enter (i.e., $\mathrm{HgCl}_{2}$ ), but this possibility cannot be demonstrated in our conditions.

\section{References}

[1] D.W. Boening, Chemosphere 40 (2000) 1335. 
[2] M.W. Smith, P.C. Phelps, H.D. Humes, J. Biol. Chem. 88 (1991) 4926.

[3] M. Konigsberg, N.E. Lopez-Diazguerriero, L. Bucio, M.C. Gutierrez-Ruiz, J. Appl. Toxicol. 21 (2001) 323.

[4] B.C. Kone, R.M. Brenner, S.R. Gullans, J. Membr. Biol. 113 (1990) 1.

[5] E.R. Stadtman, C.N. Oliviers, J. Biol. Chem. 266 (1991) 2005.

[6] L.P. Karniski, J. Biol. Chem. 267 (1992) 19218.

[7] P.L. Savant, S. Shibko, U.S. Kumta, A.L. Tappel, Biochim. Biophys. Acta 85 (1964) 82.

[8] O.H. Lowry, N.J. Rosenbrough, D.L. Farr, R.J. Randall, J. Biol. Chem. 193 (1951) 265

[9] Y. Muriyama, M. Maeda, M. Futai, FEBS Lett. 302 (1992) 18.
[10] S. Ohkuma, Y. Moriyama, T. Takano, J. Biochem. 94 (1983) 1935.

[11] I. Mellman, R. Fuchs, A. Helenius, Ann. Rev. Biochem. 55 (1986) 663.

[12] P.O. Segeln, Method Enzymol. 96 (1993) 737.

[13] P. Dell'Antone, M. Bragadin, P. Zatta, Biochim. Biophys. Acta 1270 (1995) 137.

[14] D. Kleiner, Biochim. Biophys. Acta 639 (1981) 41.

[15] P. Dell'Antone, R. Colonna, G.F. Azzone, Eur. J. Biochem. 24 (1972) 553.

[16] E.J. Bowman, A. Siebers, K. Altendorf, Proc. Natl. Acad. Sci. USA 85 (1988) 7972.

[17] P. Mitchell, Science 206 (1979) 1148. 